\title{
Dual-Band Compact Metamaterial-Inspired Absorber with Wide Incidence Angle and Polarization Insensitivity for GSM and ISM Band Applications
}

\author{
Kanwar Preet KAUR ${ }^{1}$, Trushit UPADHYAYA ${ }^{1}$, Merih PALANDOKEN ${ }^{2}$ \\ ${ }^{1}$ Dept. of Electronics and Communication Engineering, Chandubhai S. Patel Inst. of Technology, \\ Charotar University of Science and Technology, 38842-Changa, Anand (Gujarat), India \\ ${ }^{2}$ Dept. of Electrical and Electronic Engineering, Izmir Katip Celebi University, 35620 Izmir, Turkey \\ kanwarpreet27@gmail.com, trushitupadhyaya.ec@charusat.ac.in, merih.palandoken@ikc.edu.tr \\ Submitted November 11, 2017 / Accepted July 17, 2018
}

\begin{abstract}
A dual-band metamaterial inspired microwave absorber composed of concentric two crossed double-arrow shaped resonators, ring resonator with four splits at the corners and square ring resonator is presented. The proposed RF absorber has the absorption feature of wide incidence angle. The sub-wavelength unit cell of the proposed absorber is structured on a metal backed epoxy glass (FR-4) substrate. The novel absorber has two distinct absorption peaks of $99.4 \%$ and $98.6 \%$ at the frequencies of $1.94 \mathrm{GHz}$ and $2.4 \mathrm{GHz}$, respectively. The designed structure is polarization-insensitive with wide incidence angle of $60^{\circ}$ and high absorption rate of $82 \%$ for transverse electric and $98 \%$ for transverse magnetic modes. Polarization insensitivity of the proposed design is investigated by the waveguide measurement technique with setting different orientation angles for the unit cells. The measured and simulated results have good agreement making the proposed absorber a potential candidate for energy harvesting applications in GSM and ISM band.
\end{abstract}

\section{Keywords}

Metamaterials, metamaterial absorber, ring resonators, polarization

\section{Introduction}

Metamaterials (MTMs) are artificial materials whose electric and magnetic responses can be altered according to the required application to exhibit exotic properties not usually available in nature [1], [2]. Among several MTM based RF component applications like antennas [3-5], filters [6], [7] and high frequency photodiode bias Tee circuits [8], metamaterial absorbers (MMA) [9] are extensively proposed and investigated due to the various properties exhibited in the form of multi-band [10], polarization independence [11], compact [12], broad-band [13], [14], and wide-oblique angle [15], [16]. These exotic character- istics of MMAs are achieved merely by altering the geometrical parameters of the sub-wavelength unit cells which are usually arranged in a periodic manner. The variation is done to achieve impedance matching between the impedance of the MMA structure and the free space impedance resulting in minimization of the electromagnetic (EM) reflection from the MMA surface.

In this article, a compact dual-band polarization insensitive MMA with wide incidence angle is designed and developed for trapping of ambient GSM and ISM band EM signals. The suggested absorber exhibits two distinct absorption peaks of $99.4 \%$ and $98.6 \%$ with the corresponding return losses of $22.35 \mathrm{~dB}$ and $18.43 \mathrm{~dB}$ at the frequencies of $1.94 \mathrm{GHz}$ and $2.4 \mathrm{GHz}$, respectively. The FWHM (full width at half maxima) bandwidth of the both absorption bands are in the range of $4 \%$. The narrow bandwidth can be increased by increasing the substrate height or utilizing more intercoupled resonators with the enhanced electric and magnetic coupling level. To the extent of author's knowledge, no microwave MMA has been reported so far for the aforementioned frequency band and the proposed absorber is also tested for the polarization insensitivity using waveguide measurement technique [13-15]. This is mainly because of the necessity of eliminating large sample size $(\sim 10 \lambda)$ requirement for the selected frequency band in case of free-space measurement system [8]. Polarization angle and oblique angle variations for both TE (transverse electric) and TM (transverse magnetic) modes are illustrated to show that the proposed absorber is polarization insensitive with fairly high absorption of $82 \%$ and $98 \%$, respectively, for wide oblique incidence angle of about $60^{\circ}$. The waveguide measurement results for polarization insensitivity of the suggested design are presented with good agreement with the numerical results. The proposed unit cell design is compact and ultrathin with size and thickness of $0.17 \lambda_{0}$ and $0.015 \lambda_{0}$, respectively, at the lowest frequency of absorption.

Dimension of MMA must be selected such that it is less or equal to $\lambda / 4$ ( $\lambda$ is the operating wavelength wave- 
length) [17]. The lower the operating frequency the larger is the unit cell size and vice versa. The fabricated sample has arrays of unit cells arranged in the periodic manner. Hence, to maintain the moderate size of the fabricated sample the electrical length of resonators must be increased. Resonator shape is selected such that it has folding symmetry in order to get polarization stability. Most widely reported polarization insensitive MMA includes concentric closed ring resonator (CRR) [10] structures because of their folding symmetry. Other resonators that can be used are split ring resonators (SRRs) and electric ring resonators (ERRs) [9]. The thickness of the substrate is determined by the required application. The larger the substrate thickness the more is the bandwidth and the lower is the absorption.

\section{Proposed Absorber Design}

The unit cell of the proposed wide angle absorber consists of three resonator structures. These are two crossed double-arrow shaped resonators, ring resonator with four splits at the corners and square ring resonator. The first step of designing MMA is to choose the operating frequency. In this article operating frequency is selected as $1.94 \mathrm{GHz}$. Corresponding to this operating frequency initial size of the unit cell is set as $38 \mathrm{~mm}$ as it is less than $\lambda / 4$, the required condition for the unit cell to operate as MTM and substrate height is selected as $2.4 \mathrm{~mm}$ because resonant absorbers have narrower bandwidth. The next step is to design a unique resonator structure for achieving multiband resonance depending upon the required application and should be independent to polarization. With $38 \mathrm{~mm}$ unit cell size the absorption frequency obtained is very less

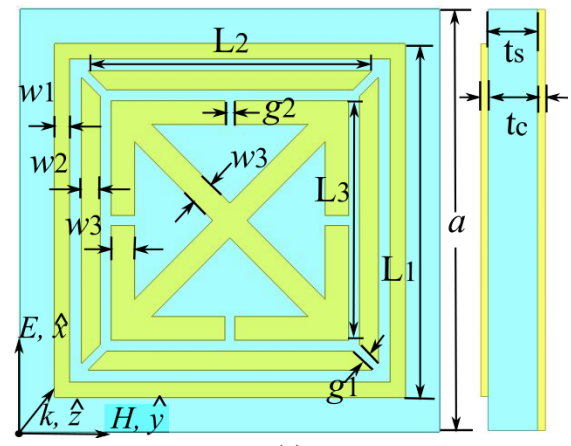

(a)

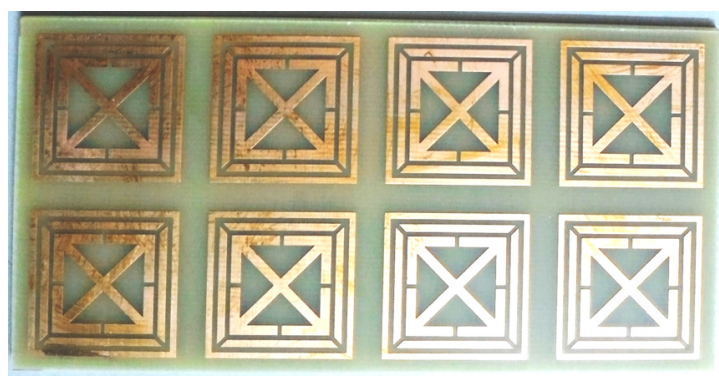

(b)

Fig. 1. The proposed wide angle absorber: (a) Unit cell with geometrical dimensions and (b) fabricated $2 \times 4$ array MMA prototype. compared to the target frequency. Therefore, the unit cell of the proposed absorber is further reduced such that absorption band is obtained at target frequency. Also, considering that the fabricated sample has arrays of unit cells arranged in the periodic manner, the unit cell size is reduced to accommodate more number of unit cells in the eventual MMA sample. With this design procedure any MMA can be designed.

The proposed MMA unit cell model is shown in Fig. 1(a) along with the fabricated $2 \times 4$ array MMA prototype in Fig. 1(b). The design is engraved on a metal backed epoxy glass (FR-4) substrate of height $\left(t_{\mathrm{s}}\right) 2.4 \mathrm{~mm}$, relative dielectric constant of 4.4 and loss tangent $(\tan \delta)$ of 0.02 . The thickness of metal conductor $\left(t_{\mathrm{c}}\right)$ is selected as $0.035 \mathrm{~mm}$, much greater than the skin depth to suppress any transmission through the absorber, and has electric conductivity $(\sigma)$ of $5.8 \times 10^{7} \mathrm{~S} / \mathrm{m}$. The optimized geometrical parameters are as follows: $a=27.4, L_{1}=22.9, L_{2}=$ $18.5, L_{3}=15.56, w_{1}=0.99, w_{2}=1.2, w_{3}=w_{4}=1.56$ and $g_{1}$ $=g_{2}=0.6$. All dimensions are in millimeters $(\mathrm{mm})$. The physical size of MMA unit cell is $0.17 \lambda_{0} \times 0.17 \lambda_{0}$, where $\lambda_{0}$ is the free space wavelength at the lowest absorption frequency.

\section{Numerical Computation Results}

To obtain the numerical RF characterization of the proposed absorber, commercial 3D electromagnetic field solver, HFSS has been utilized with the open boundary conditions along the direction of propagation and periodic boundary conditions in the lateral directions. By determining the reflection coefficient, $S_{11}(\omega)$, and the transmission coefficient, $S_{21}(\omega)$, of the proposed MMA various absorber parameters are obtained by using equations (1) through (4). The RF performance of the proposed absorber is illustrated in Fig. 2 with dual absorption maxima of $99.4 \%$ and $98.6 \%$ at $1.94 \mathrm{GHz}$ and $2.4 \mathrm{GHz}$, respectively. The outer ring and the center split ring causes the lower absorption band whereas the upper absorption band is generated by the inner most crossed double-arrow shaped resonators and center split ring.

The proposed wide angle absorber is analyzed for the effect of presence of individual resonator in Fig. 3. It is evident that individual resonator structure is responsible for absorption at a single frequency except for structure II. Structure I and structure II show high absorption of $98.17 \%$ and $97.38 \%$ at $1.9217 \mathrm{GHz}$ and $2.552 \mathrm{GHz}$, respectively, whereas structure III exhibits absorption of $78.44 \%$ at $2.32 \mathrm{GHz}$. The combination of three structures results in frequency conversion due to mutual coupling between the structures which assist in achieving absorption response at significant frequency of $2.4 \mathrm{GHz}$.

The presented absorber is numerically simulated with various polarization angles $(\phi)$ at normal incidence to confirm the polarization independence of the absorber structure. Figure 4 shows the polarization insensitivity of the 


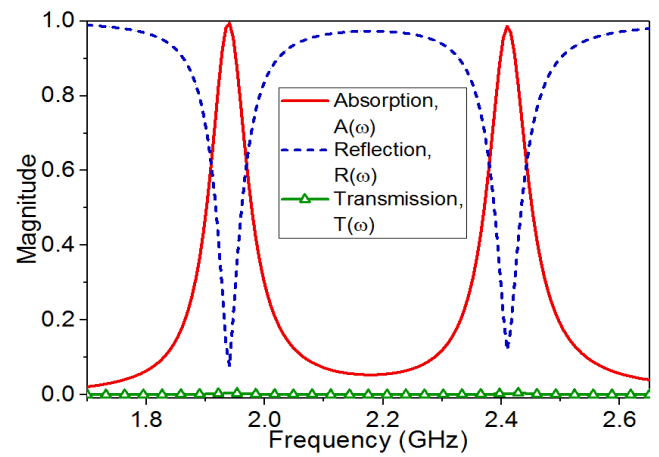

Fig. 2. Numerical computation results for RF transmission, reflection and absorption characteristics.

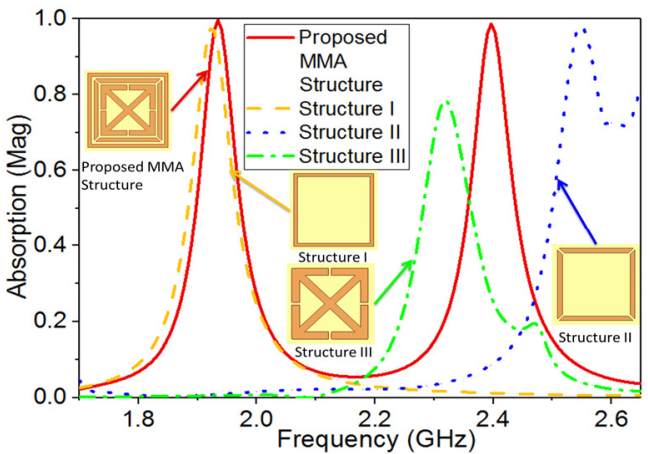

Fig. 3. Numerical computation results depicting comparison of absorption response for different design structures.

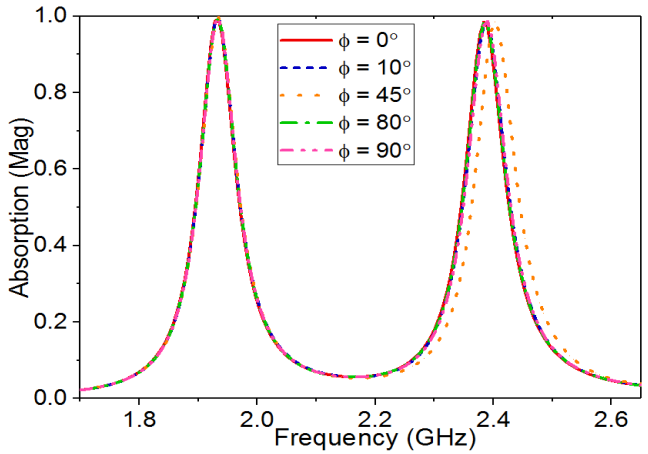

Fig. 4. Numerically simulated absorption response at various polarization angles $(\phi)$ under normal incidence.

absorber structure, which is the consequence of the fourfold design symmetry. Moreover, the proposed wide angle absorber is also studied for the oblique incidence $(\theta)$ of EM wave under TE and TM mode configuration as illustrated in Figs. 5(a) and 5(b), respectively. The $\theta$ angle variation graph depicts that the absorber design exhibits high absorption of about $82 \%$ for TE mode and above $98 \%$ for TM mode up to $60^{\circ}$ of $\theta$ angle.

\section{Absorption Theory}

The absorption principle of MMA depends on the effective medium parameters. Perfect absorption $(100 \%$ or unity absorption) is achieved by individually regulating effective electric permittivity $\varepsilon_{\text {eff }}(\omega)$ and effective magnetic permeability $\mu_{\mathrm{eff}}(\omega)$ of the homogeneous structure. The

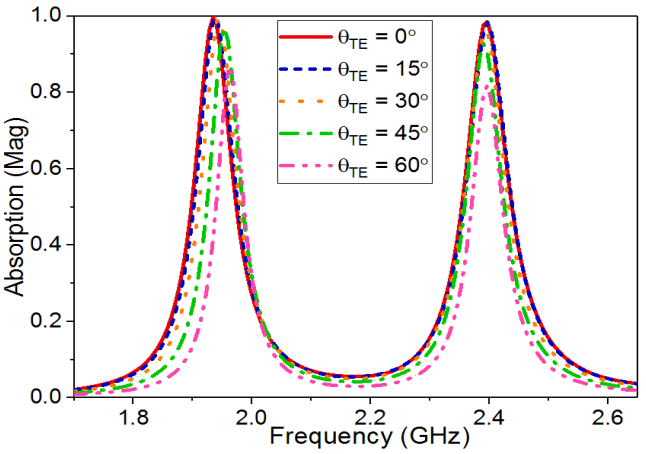

(a)

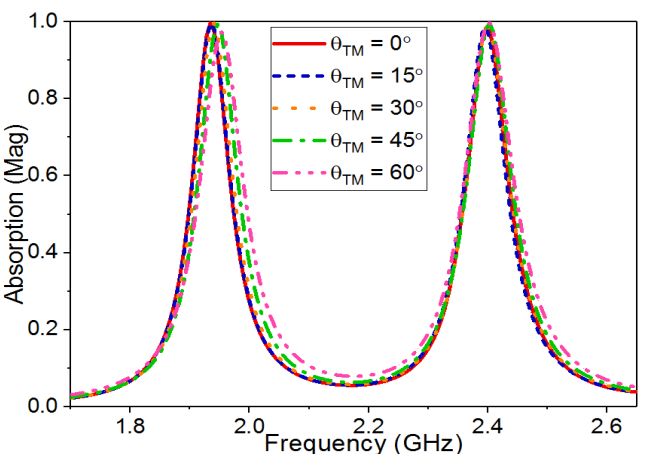

(b)

Fig. 5. Numerically simulated results of absorption at various oblique angles $(\theta)$ for: (a) TE mode and (b) TM mode.

absorption efficiency is the key parameter of MMA and is defined by the equation

$$
A(\omega)=1-R(\omega)-T(\omega)
$$

where $A(\omega)$ is the absorption coefficient, $R(\omega)$ and $T(\omega)$ are respectively the reflectance and transmission. $R(\omega)$ and $T(\omega)$ are related to the reflection coefficient and the transmission coefficient in the manner: $\left|S_{11}(\omega)\right|^{2}$ and $\left|S_{21}(\omega)\right|^{2}$, respectively.

Thus, equation (1) can be rewritten as

$$
A(\omega)=1-\left|S_{11}(\omega)\right|^{2}-\left|S_{21}(\omega)\right|^{2} .
$$

Unity absorption is achieved by reducing both $R(\omega)$ and $T(\omega)$ to zero. $T(\omega)$ is reduced to zero theoretically by placing a metal ground plate at the bottom side having thickness much greater than its skin depth at the guided wavelength thereby reducing equation (2) as

$$
A(\omega)=1-\left|S_{11}(\omega)\right|^{2} .
$$

However, $R(\omega)$ cannot be minimized to perfect zero as its reduction depends on the matching of the structure's impedance to the free-space impedance. This impedance matching causes real component of the effective normalized impedance of the structure to approach unity while imaginary component approaches zero making real components and imaginary components of $\varepsilon_{\mathrm{eff}}(\omega)$ and $\mu_{\mathrm{eff}}(\omega)$ approximately equal. The normalized effective impedance of the wide angle absorber depicted in Fig. 6 is extracted from the following equation [17] 


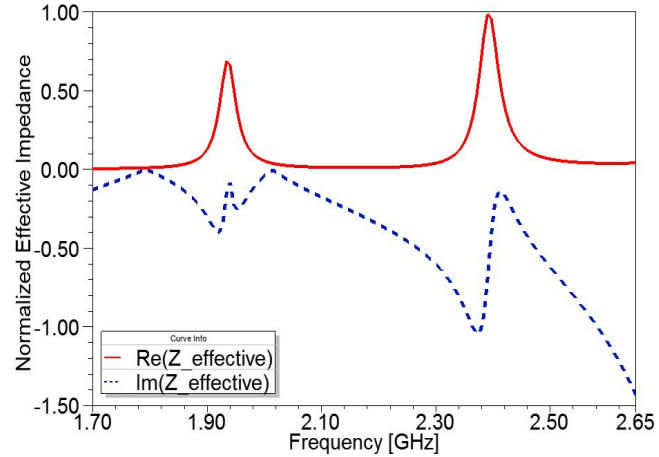

Fig. 6. Retrieved normalized effective impedance of the proposed wide angle absorber.

$$
Z(\omega)=\sqrt{\frac{\left(1+S_{11}(\omega)\right)^{2}-S_{21}^{2}(\omega)}{\left(1-S_{11}(\omega)\right)^{2}-S_{21}^{2}(\omega)}} .
$$

To get into the physics behind the absorption using metamaterial technique the surface current distribution and the electric field distribution are plotted for both the absorption band frequencies. Figures 7 and 8 depict the antiparallel surface current flowing in the top and bottom metal conductor at 1.94 and $2.4 \mathrm{GHz}$, respectively. It is apparent that the surface current directions on top face and bottom face are anti-parallel with each other at the target frequencies. These anti-parallel currents create circulating current loops in perpendicular direction about incident magnetic field, thus, generating magnetic excitations which influence the $\mu_{\mathrm{eff}}(\omega)$ of the array structure.

The induced electric field distribution plots for $1.94 \mathrm{GHz}$ and $2.4 \mathrm{GHz}$ are depicted in Fig. 9(a) and Fig. 9(b), respectively. The $\varepsilon_{\text {eff }}(\omega)$ is controlled by induced electric excitation through incident electric field on the top face of the structure. It is observed that the strong concentration of the electric field at the outer most ring creates the first resonance at $1.94 \mathrm{GHz}$ whereas electric field concentration in the inner most crossed double-arrow shaped resonators ring is responsible for the resonance at $2.4 \mathrm{GHz}$. The center split ring contributes to both the band. By changing the gaps between the rings and by altering width as well as length modifies the effective capacitances and inductance hence altering $\varepsilon_{\text {eff }}(\omega)$ and $\mu_{\text {eff }}(\omega)$ to consequently results in desired absorption and respective frequency shift.

Furthermore, the sensitivity of the proposed wide angle absorber to the incident power is investigated by determining the net power flow through the top surface of the proposed absorber. This is achieved by integrating the Poynting vector normal to that surface.

$$
W=\int_{S} \operatorname{Re}(\boldsymbol{P}) \cdot \boldsymbol{n} \mathrm{d} S
$$

where S represents the top surface of the proposed MMA and $\boldsymbol{n}$ is the normal vector to the surface S. It is observed from Fig. 10 that the proposed MMA absorber structure is less sensitive to the incident power as the amount of power

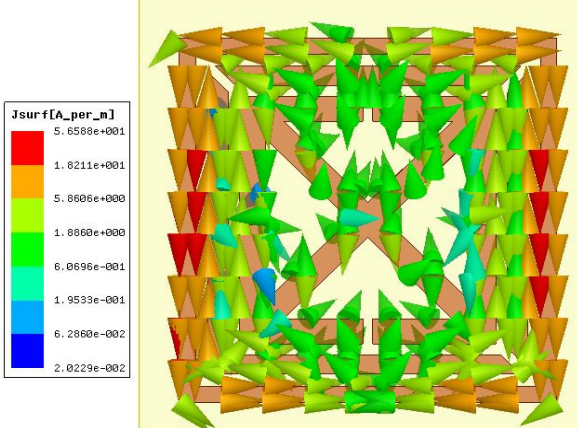

(a)

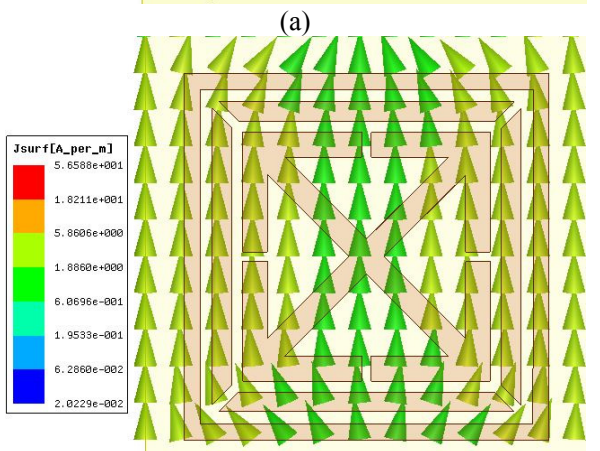

(b)

Fig. 7. Current density distribution at $1.94 \mathrm{GHz}$ : (a) Top face and (b) bottom face.

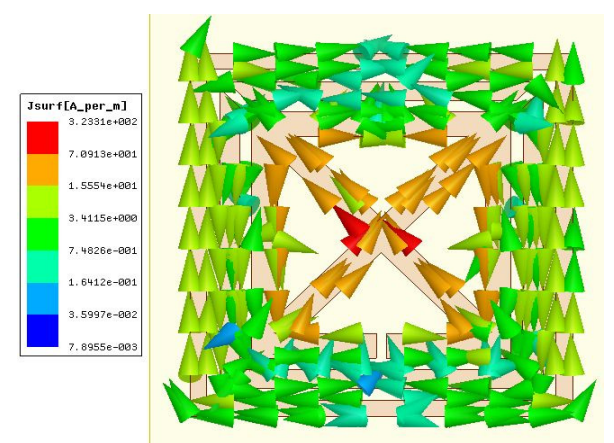

(a)

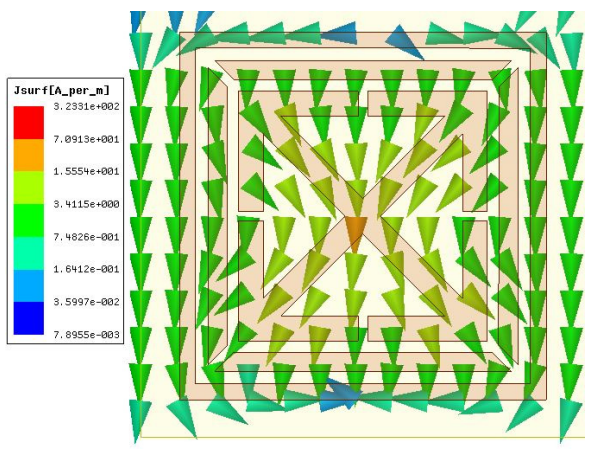

(b)

Fig. 8. Current density distribution at $2.4 \mathrm{GHz}$ : (a) Top face and (b) bottom face.

incident is approximately equals the power absorbed. Additionally, Table 1 provides the power absorption by individual structures in comparison to the proposed MMA. It is observed from Tab. 1 that the proposed MMA has got absorbed power response superior to other structures. Among three structures, structure I absorbs maximum 
incident power which is also seen from the electric field distribution plot, Fig. 9.

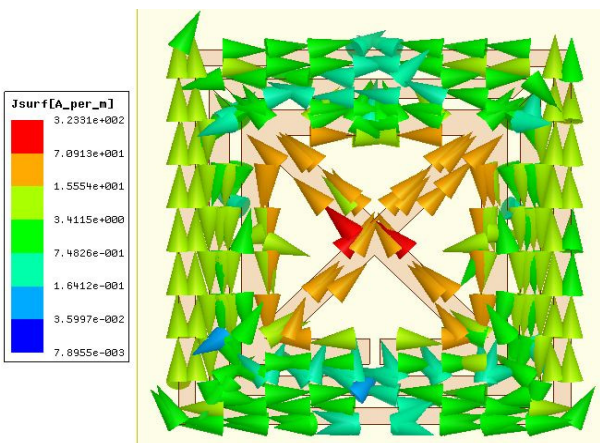

(a)

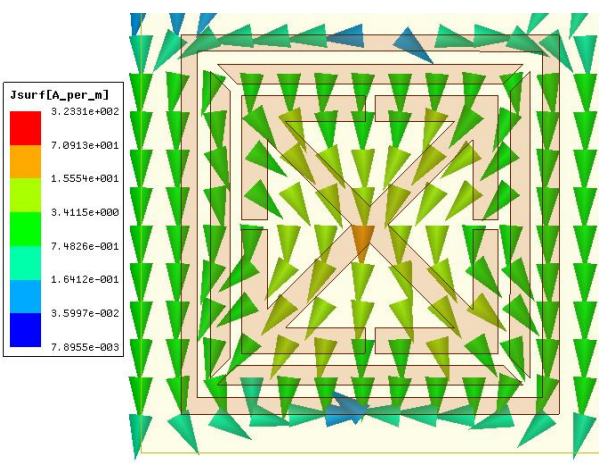

(b)

Fig. 8. Current density distribution at $2.4 \mathrm{GHz}$ : (a) Top face and (b) bottom face.

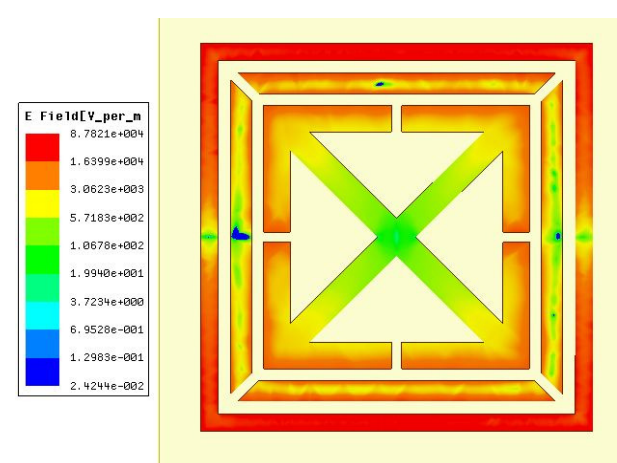

(a)

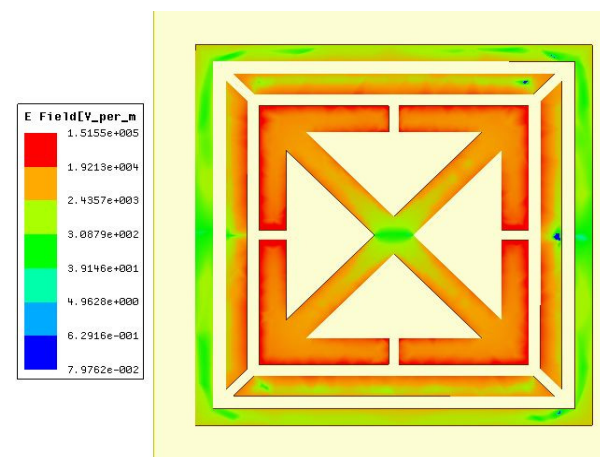

(b)

Fig. 9. Induced electric field distribution for (a) $1.94 \mathrm{GHz}$ and (b) $2.4 \mathrm{GHz}$

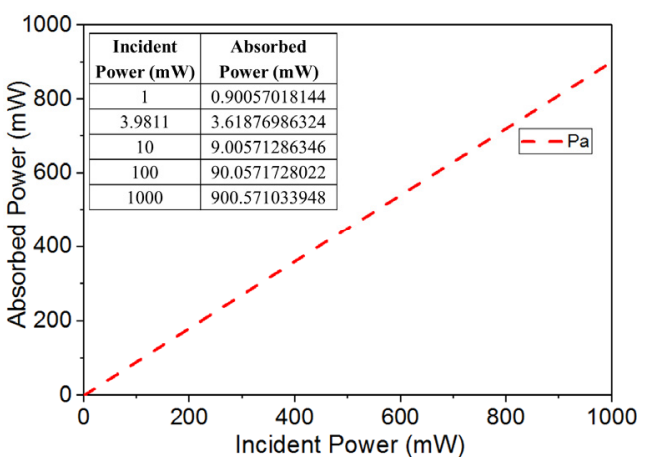

Fig. 10. Numerically simulated results for investigating power sensitivity of proposed MMA.

\begin{tabular}{|l|l|}
\hline Structure & Power Absorbed (W) \\
\hline Proposed MMA & 0.90057103394795 \\
\hline Structure I & 0.875164444688426 \\
\hline Structure II & 0.00596058976515394 \\
\hline Structure III & 0.00493210431572523 \\
\hline
\end{tabular}

Tab. 1. Power absorbed by individual structure in comparison with the proposed MMA for $1 \mathrm{~W}$ of incident power.

\section{Experimental Setup and Results}

The proposed wide-angle absorber is tested using waveguide measurement method. A standard WR-430 waveguide is connected to Agilent N9912A vector network analyzer (VNA) via Teflon coaxial cable. The proposed absorber sample has the same geometrical size as that of the inner aperture dimension of the waveguide. The measurement setup is shown in Fig. 11. The proposed absorber has been experimentally verified for the polarization insensitivity by setting different polarization orientation angles. As seen from Fig. 4, the proposed absorber design is polarization insensitive with the slight frequency shift in the higher absorption band for $45^{\circ}$.

For practical investigation of polarization insensitivity, the following set of orientation angles is selected for fabricating the unit cell samples: $10^{\circ}$ and $80^{\circ}$, where MMA sample of $0^{\circ}$ orientation angle has been fabricated as shown in Fig. 1(b). The samples are fabricated using standard printed circuit board (PCB) technique and are shown in Fig. 12. The measured results of the reflection coefficient for various orientations are depicted in Fig. 13. As depicted in Fig. 13, the dual-band absorber resonance frequencies are obtained at $1.9 \mathrm{GHz}$ and $2.36 \mathrm{GHz}, 1.89 \mathrm{GHz}$ and $2.37 \mathrm{GHz}$, and $1.91 \mathrm{GHz}$ and $2.37 \mathrm{GHz}$ frequencies for orientations of $0^{\circ}, 10^{\circ}$ and $80^{\circ}$, respectively.

The corresponding absorption graphs compared with $0^{\circ}$ numerical result are illustrated in Fig. 14, ensuring that the absorption rate of the proposed wide angle MMA is independent of polarization angle variation. The $0^{\circ}$ incidence angle of TE mode excited incoming wave in waveguide method [14] has caused approximately $0.03 \mathrm{GHz}-0.04 \mathrm{GHz}$ shift in absorption frequency. This frequency shift is also aided by the fabrication mismatch tolerance and material parameter changes. The comparison 
of state-of-the-art MMA absorbers with the proposed absorber is presented in Tab. 2. As deduced from Tab. 2, the proposed MMA has better absorption level in more compact cell size with novel unit cell geometry.

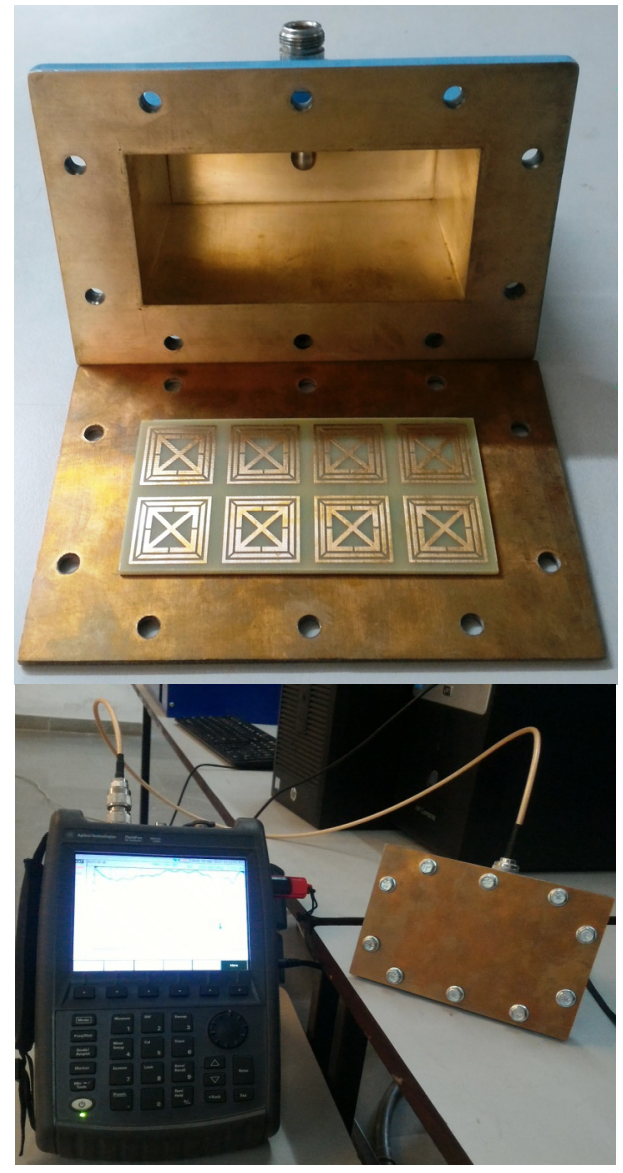

Fig. 11. Waveguide measurement setup for the proposed wide angle absorber.

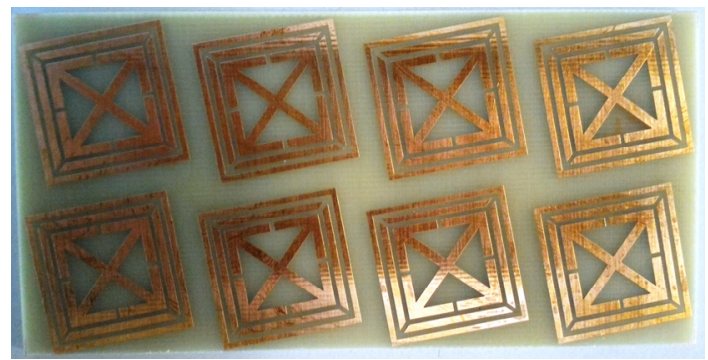

(a)

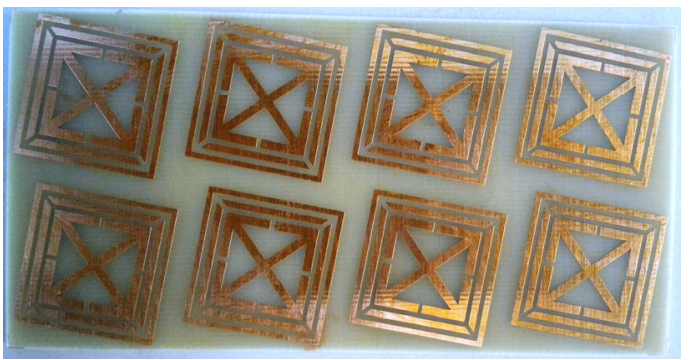

(b)

Fig. 12. Photographs of fabricated samples with orientation of (a) $10^{\circ}$ (Sample-2) and (b) $80^{\circ}$ (Sample-3).

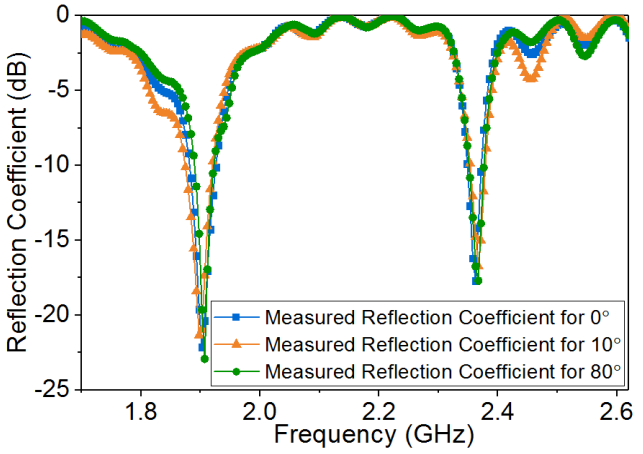

Fig. 13. Measured reflection coefficient of the proposed wide angle absorber for various orientations.

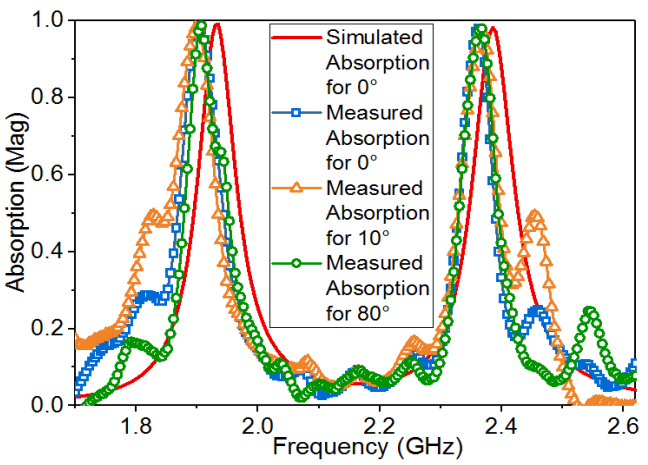

Fig. 14. Measured absorption of the proposed wide angle absorber for various orientations in comparison with numerical absorption.

\begin{tabular}{|c|c|c|c|c|}
\hline 节 & 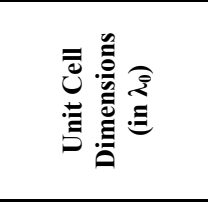 & 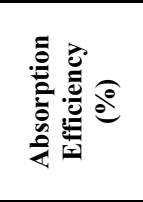 & 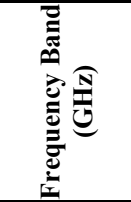 & 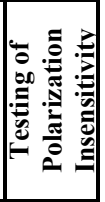 \\
\hline [5] & $0.26 \times 0.26 \times 0.083$ & $>90$ & $\begin{array}{l}\text { Broadband } \\
8.3 \text { to } 14.6 \\
\end{array}$ & No \\
\hline [6] & $0.5 \times 0.5 \times 0.125$ & $>90$ & $\begin{array}{l}\text { Wideband } \\
12.5 \text { to } 14\end{array}$ & No \\
\hline [7] & $0.2 \times 0.2 \times 0.015$ & $>95$ & $\begin{array}{l}\text { Dual band } \\
5.1 \& 8.5\end{array}$ & No \\
\hline $\begin{array}{c}\text { Proposed } \\
\text { MMA }\end{array}$ & $0.17 \times 0.17 \times 0.015$ & $99.4 \& 98.6$ & $\begin{array}{l}\text { Dual band } \\
1.94 \& 2.4\end{array}$ & Yes \\
\hline
\end{tabular}

Tab. 2. Comparison table of the proposed and state-of-the-art $\mathrm{RF}$ absorber designs.

\section{Conclusion}

A compact EM wave absorber with wide incidence angle of $60^{\circ}$ having high absorption rate of $82 \%$ for TEmode and $98 \%$ for TM-mode is presented in this article at dual frequencies using MTM inspired unit cell design. The proposed wide angle MMA is also studied for the polarization insensitivity and is practically verified using the waveguide measurement technique. The practical verification of polarization insensitivity of presented MMA is done by fabricating different samples for different orientations of unit cells. Both numerically simulated and experimentally measured results are in good agreement, making the current design a good candidate for the harvesting ambient 
GSM and ISM band energy in RF energy harvesting systems. The proposed unit cell design is compact and ultrathin at the lowest frequency of absorption with size and thickness of $0.17 \lambda_{0}$ and $0.015 \lambda_{0}$, respectively.

\section{References}

[1] VESELAGO, V. G. The electrodynamics of substances with simultaneously negative values of $\varepsilon$ and $\mu$. Soviet Physics Uspekhi, 1968, vol. 10, no. 4, p. 509-514. DOI: 10.1070/PU1968v010n04ABEH003699

[2] SHELBY, R. A., SMITH, D. R., SCHULTZ, S. Experimental verification of a negative index of refraction. Science, 2000, vol. 292 , no. 5514 , p. $77-79$. DOI: $10.1126 /$ science. 1058847

[3] PALANDÖKEN, M. Microstrip antenna with compact anti-spiral slot resonator for $2.4 \mathrm{GHz}$ energy harvesting applications. Microwave and Optical Technology Letters, 2016, vol. 58, no. 6, p. 1404-1408. DOI: 10.1002/mop.29824

[4] UPADHYAYA, T. K., KOSTA, S. P., JYOTI, R., PALANDÖKEN, M. Negative refractive index material-inspired 90-deg electrically tilted ultra wideband resonator. Optical Engineering, 2014, vol. 53, no. 10, p. 1-4. DOI: 10.1117/1.OE.53.10.107104

[5] UPADHYAYA, T. K., KOSTA, S. P., JYOTI, R., PALANDÖKEN, M. Novel stacked $\mu$-negative material-loaded antenna for satellite applications. International Journal of Microwave and Wireless Technologies, 2016, vol. 8, no. 2, p. 229-235. DOI: 10.1017/S175907871400138X

[6] PALANDÖKEN, M., SONDAS, A. Compact metamaterial based bandstop filter. Microwave Journal, 2014, vol. 57, no. 10, p. 76-84. ISSN: 0192-6225

[7] PALANDÖKEN, M., UCAR, M. H. Compact metamaterial-inspired band-pass filter. Microwave and Optical Technology Letters, 2014, vol. 56, no. 12, p. 2903-2907. DOI: $10.1002 /$ mop. 28724

[8] PALANDÖKEN, M., RYMANOV, V., STÖHR, A., TEKIN, T. Compact metamaterial-based bias Tee design for $1.55 \mu \mathrm{m}$ waveguide-photodiode based $71-76 \mathrm{GHz}$ wireless transmitter. In Progress In Electromagnetics Research Symposium. Moscow (Russia), 2012, p. 393-397. ISSN: 1559-9450

[9] LANDY, N. I., SAJUYIGBE, S., MOCK, J. J., et al. Perfect metamaterial absorber. Physical Review Letters, 2008, vol. 100, no. 20 , p. 1-4. DOI: 10.1103/PhysRevLett.100.207402

[10] SOOD, D., TRIPATHI, C. C. A polarization insensitive compact ultrathin wide-angle penta-band metamaterial absorber. Journal of Electromagnetic Waves and Applications, 2017, vol. 31, no. 4, p. 394-404. DOI: 10.1080/09205071.2017.1288172

[11] SHARMA, S. K., GHOSH, S., SRIVASTAVA, K. V., SHUKLA, A. Ultra-thin dual-band polarization-insensitive conformal metamaterial absorber. Microwave and Optical Technology Letters, 2017, vol. 59, no. 2, p. 348-353. DOI: 10.1002/mop.30285

[12] RAMYA, S., SRINIVASA RAO, I. A compact ultra-thin ultra-wideband microwave metamaterial absorber. Microwave and Optical Technology Letters, 2017, vol. 59, no. 8, p. 1837-1845. DOI: $10.1002 / \mathrm{mop} .30636$

[13] ZHAI, H., ZHANG, B., ZHANG, K., ZHAN, C. A stub-loaded reconfigurable broadband metamaterial absorber with wide-angle and polarization stability. Journal of Electromagnetic Waves and Applications, 2017, vol. 31, no. 4, p. 447-459. DOI: 10.1080/09205071.2017.1293567
[14] ZHAI, H., ZHAN, C., LIU, L., ZANG, Y. Reconfigurable wideband metamaterial absorber with wide angle and polarisation stability. Electronics Letters, 2015, vol. 51, no. 21, p. 1624-1626. DOI: $10.1049 / \mathrm{el} .2015 .1557$

[15] ZHAI, H., ZHAN, C., LIU, L., LIANG, C. A new tunable dualband metamaterial absorber with wide-angle TE and TM polarization stability. Journal of Electromagnetic Waves and Applications, 2015, vol. 29, no. 6, p. 774-785, DOI: 10.1080/09205071.2015.1024335

[16] AGARWAL, M., BEHERA, A. K., MESHRAM, M. K. Wideangle quad-band polarisation-insensitive metamaterial absorber. Electronics Letters, 2016, vol. 52, no. 5, p. 340-342. DOI: 10.1049/el.2015.4134

[17] CALOZ, C., ITOH, T. Electromagnetic Metamaterials: Transmission Line Theory and Microwave Applications. John Wiley \& Sons, 2005, p. 2. ISBN: 978-0-471-66985-2

[18] SMITH, D. R., VIER, D. C., KOSCHNY, T., SOUKOULIS, C. M Electromagnetic parameter retrieval from inhomogeneous metamaterials. Physical Review E, 2005, vol. 71, no. 3, p. 1-11. DOI: 10.1103/PhysRevE.71.036617

\section{About the Authors ...}

Kanwar Preet KAUR (corresponding author) received her B.E. degree in Electronics and Telecommunication from Christian College of Engineering \& Technology, Bhilai (C.G.), India, in 2004 and the M.E. degree in Communication System from Jabalpur Engineering College, Jabalpur (M.P.), India, in 2008. She is a student member of the Institute of Electrical and Electronics Engineers (IEEE) Engineers, India. At present, she is pursuing her Ph.D. degree with specialization in Metamaterial Microwave Absorber. Her main research area of interest is metamaterial based absorbers and applied electromagnetics.

Dr. Trushit UPADHYAYA has received his B.E. in Electronics and Communication Engineering from Gujarat University, Ahmedabad, Gujarat, India and M.E. in Telecommunications from the Institute of Telecommunication Research, University of South Australia, Adelaide, South Australia. Dr. Trushit has completed his Ph.D in Antenna System Design for Satellite Communication at Charotar University of Science and Technology, Changa, Gujarat, India. He is a member of the Institute of Electrical and Electronics Engineers (IEEE), Engineers Australia (EA), and the Indian Society for Technical Education (ISTE).

Merih PALANDÖKEN was born in Kahramanmaras, Turkey. He received his M.Sc. degree in the field of Microsystem and Microelectronics Engineering from Technische Universitaet Hamburg, Germany in 2005 and Ph.D. degree in the field of Metamaterial Based Antennas and Microwave Filters from the Department of Theoretical Electrical Engineering at Technische Universitaet Berlin, Germany in 2012. His research interests include bioimplantable antenna design, RF energy harvesting system design, electromagnetic field theory and electrically small antenna design. 